\title{
Flexicurity - Gibt es ein Gleichgewicht zwischen Arbeitsmarktflexibilität und sozialer Sicherheit?
}

\author{
Hartmut Seifert \\ Andranik Tangian
}

Flexicurity bezeichnet eine neue politische Strategie der Europäischen Kommission. Sie soll gleichgewichtig die Flexibilität des Arbeitsmarktes und die soziale Sicherheit fördern. Von diesem Ziel ist die Wirklichkeit jedoch noch weit entfernt. Wie die nachfolgende empirische Untersuchung für die 27 Mitgliedsländer der Europäischen Union (EU) zeigt, besteht eher ein negativer Zusammenhang zwischen beiden Komponenten. Gefordert ist deshalb eine Flexicurity-Strategie, deren Hauptaugenmerk auf die Verbesserung der sozialen Sicherheit gerichtet ist.

\section{Problemstellung}

Mit dem Grünbuch „Ein modernes Arbeitsrecht für die Herausforderung des 21. Jahrhunderts" (European Commission 2006a) hat die Europäische Kommission eine Debatte über Flexicurity initiiert. Diese vor allem in den "gemeinsamen Grundsätzen" (European Commission 2007a) weiter entwickelten Überlegungen zielen nicht nur auf die Reform der rechtlichinstitutionellen Rahmenbedingungen des Arbeitsmarktes. Gleichzeitig und gleichgewichtig sollen sie auch die Beschäftigungssicherheit erhöhen. Der Flexicurity-Ansatz soll außerdem Flexibilitätsanforderungen und -bedürfnissen der Beschäftigten Rechnung tragen. Die Quadratur des Kreises? Auf jeden Fall ist dieser Vorstoß als implizites Eingeständnis zu werten, dass vermehrte Flexibilität am Arbeitsmarkt nicht automatisch auch die soziale Sicherheit verbessert. Gesucht ist deshalb eine neue Politikstrategie, die nicht wie bisher allein auf Flexibilisierung setzt und hofft, das möglichst freie Spiel der Marktkräfte würde auch für soziale Sicherheit sorgen.

Allenfalls vage lassen die "gemeinsamen Grundsätze“ erkennen, wie das neue Konzept aussehen soll. Vorgeschlagen werden dort unter anderem „moderne Systeme der sozialen Sicherheit“, „umfassende Strategien des lebenslangen Lernens" und „wirksame aktive arbeitsmarktpolitische Maßnahmen" (European Commission 2007a), ohne diese Punkte jedoch konzeptionell und instrumentell zu präzisieren. Die EU-Kommission überlässt es den Mitgliedsländern, Flexicurity-Konzepte zu entwickeln, die auf deren spezifische Problem- lagen zugeschnitten sind und zu den jeweiligen institutionellen Rahmenbedingungen passen. $\mathrm{Zu}$ unterschiedlich sind die Ausgangslagen in den Mitgliedsländern, als dass ein einheitliches Flexicurity-Konzept Erfolgschancen hätte. Immerhin steht das skizzierte Zieldreieck für einen bemerkenswerten Richtungswechsel. Die Kommission korrigiert ihre bisherige Linie, die auf eine Strategie der reinen Flexibilisierung setzte und dabei die Beschäftigtenperspektive ausblendete.

Die bisherigen Flexicurity-Überlegungen leiden nicht nur an konzeptionellen Schwächen (Keller/Seifert 2008), sie sind auch empirisch und theoretisch unzureichend fundiert. Bisherige Analysen leuchten nur ausschnitthaft aus, in welchem Verhältnis Flexibilität und soziale Sicherheit zueinander stehen (European Commission 2006b; European Commission 2007b). Es fehlen Untersuchungen, die zeigen, in welchen Relationen sich die beiden Komponenten von Flexicurity in den Mitgliedsländern der EU zueinander bewegen, welche Wirkungszusammenhänge zwischen den Dimensionen der Flexibilität und denen der sozialen Sicherheit bestehen. Eine solche Analyse ist aber Voraussetzung, um Ungleichgewichte und Wirkungszusammenhänge identifizieren, soziale Risiken in Folge von Deregulierungen und Flexibilisierungen erkennen und gezielte Flexicurity-Strategien entwickeln zu können.

Diesen Fragen geht der nachfolgende Beitrag nach. ${ }^{*}$ Er analysiert den Zusammenhang von Flexibilität und sozialer Sicherheit mit Hilfe von Indikatoren, die auf Daten der Vierten Europäischen Umfrage über Arbeitsbedingungen der Europäischen Stiftung für die 27 EU-Mitgliedsländer basieren (European Foundation 2007a). Mit diesem empirischen Ansatz betreten wir Neuland und können deswegen noch nicht erwarten, Erklärungen für die vorgefundenen Zusammenhänge zu liefern. Diese Aufgabe muss zukünftigen Forschungsarbeiten überlassen bleiben. Der Beitrag definiert und operationalisiert zunächst die beiden Komponenten von Flexicurity: Flexibilität und soziale Sicherheit (Abschnitt 2). Anschließend werden die Daten und der methodische Ansatz der empirischen Analyse beschrieben (Abschnitt 3), bevor die wichtigsten Ergebnisse vorgestellt und kommentiert werden (Abschnitt 4). Einige Schlussfolgerungen runden den Beitrag ab (Abschnitt 5).

\section{Die beiden Flexicurity- Komponenten}

Die empirische Analyse von Flexibilität und Beschäftigungssicherheit setzt voraus, begrifflich klar fassen zu können, was un-

Wir danken zwei anonymen Gutachtern für wertvolle Hinweise.

Hartmut Seifert, Dr., Leiter der Abteilung WSI in der Hans-Böckler-Stiftung. Arbeitsschwerpunkte: Arbeitszeitpolitik und Arbeitsmarktforschung. e-mail: hartmut-seifert@boeckler.de Andranik Tangian, Dr. Dr., Wissenschaftler im WSI in der Hans-Böckler-Stiftung. PD Univ. Karlsruhe. Arbeitsschwerpunkte: Policy modelling und Entwicklung neuer sozioökonomischer Indikatoren. e-mail: andranik-tangian@boeckler.de 
ter diesen beiden Komponenten des Flexicurity-Ansatzes zu verstehen ist. Jedoch: Der Untersuchungsgegenstand ist in der wissenschaftlichen und politischen Diskussion nicht eindeutig definiert. Wir müssen uns folglich dem Verständnis von Flexibilität und Beschäftigungssicherheit induktiv nähern.

\subsection{FLEXIBILITÄT}

In der beschäftigungspolitischen Debatte wurde der Begriff Flexibilität lange Zeit eher unspezifisch gebraucht, ohne zwischen Formen bzw. Dimensionen und deren Funktionen systematisch zu unterscheiden. Geht man in Anlehnung an Segmentationstheorien (Edwards et al. 1973) davon aus, dass der Arbeitsmarkt aus Teilarbeitsmärkten mit unterschiedlichen Funktionslogiken und Allokationsmechanismen besteht, ist es angebracht, Formen und Strategien der Flexibilisierung zu unterscheiden. Sie beeinflussen den jeweiligen Grad der Beschäftigungssicherung und darüber hinaus auch den der sozialen Sicherung. Für eine Differenzierung sprechen auch unterschiedliche Problemkonstellationen wie kurzfristige Auftragsspitzen, saisonale oder konjunkturelle Schwankungen oder sektorale Anpassungskrisen. Sie stellen unterschiedliche Anforderungen an Anpassungsflexibilität und verlangen nach entsprechend differenzierten Strategien. Gezielte Reaktionen sind nur möglich, wenn die gesamte Palette der Flexibilisierungsformen in das anpassungsstrategische Kalkül einbezogen wird.

Eine grundlegende Unterscheidung liefert eine u. a. von der OECD verwandte Typologie (Atkinson 1984; OECD 1986), die zwischen den Hauptdimensionen interner und externer Flexibilität differenziert und diese weiter auffächert (Keller/ Seifert 2007). ${ }^{1}$ Mit der ersten Dimension interner Flexibilität sind sämtliche Strategien gemeint, die eine Anpassung des Arbeitskräfteeinsatzes an veränderte Nachfragebedingungen ohne Rückgriff auf den externen Arbeitsmarkt ermöglichen. Hierzu gehört vor allem die Anpassung der Dauer der Arbeitszeit (numerische Flexibilität), des Einkommens (monetäre Flexibilität), der Arbeitsorganisation und der Qualifikation (funktionale Flexibilität). ${ }^{2}$ Demgegenüber basiert die zweite Dimension externer Flexibilität auf der Anpassung der Beschäftigtenzahl durch Entlassungen und Einstellungen sowie dem Ein- satz von befristeten oder Leiharbeitsverhältnissen.

Eine analoge Differenzierung nimmt auch die EU vor, die sich in ihren empirischen Analysen jedoch entweder auf die Dimension der externen Flexibilität (European Commission 2006b) oder auf ausgewählte Dimensionen interner Flexibilität (European Commission 2007b) beschränkt, ${ }^{3}$ aber keinen Indikator bildet, der alle Dimensionen einschließt. Mit diesen Arbeiten knüpft die EU-Kommission an die von der OECD $(1999 ; 2004)$ durchgeführten Analysen über den Grad der Arbeitsmarktflexibilität an, erweitert jedoch die analytische Perspektive um zwei Dimensionen interner Flexibilität.

Demgegenüber basieren die OECDAnalysen über Flexibilität auf einem zusammengesetzten Index für ,employment protection legislation “ (EPL). Er misst den Grad externer Flexibilität anhand des rechtlichen Regelungsrahmens von Kündigungsschutz, Befristungen und Leiharbeit. Interne Varianten der Flexibilität bleiben ausgeblendet. Da zwischen den Dimensionen der Arbeitsmarktflexibilität sowohl substitutive als auch komplementäre Beziehungen bestehen, lässt sich der gesamte Flexibilitätsgrad nur erfassen, wenn sämtliche Flexibilitätsdimensionen berücksichtigt werden. Außerdem behandelt der EPLIndikator die institutionell-rechtlichen Rahmenbedingungen, nicht aber die faktischen Flexibilitätsgrade, deren Analyse zu wesentlich anderen Ergebnissen führen kann, wie noch zu zeigen sein wird (Abschnitt 4).

\subsection{SOZIALE SICHERHEIT - PREKARITÄT}

In ihren Publikationen zu Flexicurity unterscheidet die EU-Kommission zwischen Job- und Employment-Security, wobei sie in ihren strategischen Überlegungen ausdrücklich für einen perspektivischen Wechsel von der ersten zur zweiten Variante plädiert. Während Job-Security die Kontinuität eines spezifischen Arbeitsplatzes meint, geht es bei Employment-Security um die Kontinuität von Beschäftigungsverhältnissen, ungeachtet wechselnder Tätigkeiten und Arbeitgeber. Dieser Begriffswechsel erscheint angemessen, weil er dynamischen Aspekten Rechnung trägt und Anforderungen des Strukturwandels berücksichtigt. Gleichwohl greift er im Hinblick auf die sozialen Risiken von Flexibilität zu kurz. Employment-Security blen- det den Einkommensaspekt sowie den Schutz durch die sozialen Sicherungssysteme aus. Beide Aspekte, die Höhe des Erwerbseinkommens und dessen soziale Absicherung gegenüber den Risiken bei Krankheit, Arbeitslosigkeit und im Alter, spielen eine zentrale Rolle für die Lebensbedingungen nicht nur während, sondern auch nach der Erwerbsphase. Deshalb wird hier anstelle des von der EU-Kommission verwendeten Begriffes der Beschäftigungssicherheit dem breiteren Ansatz der sozialen Sicherheit der Vorzug gegeben.

Welche Merkmale Arbeit als sozial gesichert oder sozial ungesichert und prekär erscheinen lassen, ist in der Literatur nicht einheitlich definiert (Kraemer 2008). Je nach analytischer Perspektive variieren die zugrundegelegten Kriterien. Bei der Bewertung von atypischen Beschäftigungsverhältnissen geht ein verbreiteter Ansatz von unbefristeter Vollzeitbeschäftigung als Referenzkategorie aus und bezieht hierauf andere Beschäftigungsformen (Rodgers 1989). Prekäre Beschäftigungsverhältnisse zeichnen sich dann durch einen vergleichsweise geringen Grad an Arbeitsplatzsicherheit, eingeschränkten Schutz bzw. reduzierte Absicherung durch Gesetz oder Tarifverträge, Zugang zu den sozialen Sicherungssystemen sowie geringes Einkommen aus. Einige dieser Kriterien, wie Einkommenshöhe oder Arbeitsplatzsicherheit, lassen sich natürlich auch auf unbefristete Vollzeitarbeit beziehen. Einen ähnlichen Ansatz legt auch die EU-Kommission in Anlehnung an Arbeiten von Wilthagen/Tros (2004) in ihren Analysen zugrunde. ${ }^{4}$ Ein anderer Ansatz schließt auch subjektive und psychologische Faktoren ein (Salengros et al. 1989; Brinkmann et al. 2006).

\footnotetext{
Die Varianten können für die Funktionsfähigkeit nationaler Arbeitsmärkte je nach institutioneller Ausgestaltung unterschiedlich bedeutsam sein.

2 In der Literatur wird funktionale Flexibilität auch synonym als organisational flexibility bezeichnet (European Foundation 2003). Eine andere Unterscheidung differenziert in einer Vierfelder-Matrix zwischen interner und externer sowie quantitativer und qualitativer Flexibilität (European Foundation 2007b).

3 Die Analyse schließt Formen intern-numerischer und intern-funktionaler, nicht aber intern-monetärer Flexibilität ein.

4 Modifizierend zu den in unserer Analyse verwendeten Dimensionen führen Wilthagen/Tros (2004) noch die Dimension der "Combination security" ein, unter der die Möglichkeiten verstanden werden, bezahlte Arbeit mit anderen außerbetrieblichen Aktivitäten kombinieren zu können.
} 
Will man überprüfen, inwieweit Flexibilisierung den Grad der sozialen Sicherheit verändert und gar Prekarität entstehen lässt, sind operationalisierbare Kriterien erforderlich. Die nachfolgende Analyse stützt sich überwiegend auf objektive, aber auch auf einige subjektive Kriterien, die sich durch entsprechende Datensätze abbilden lassen:

(1) Einkommen: Das Niveau der Einkommen sollte subsistenzsichernd sein. Eine solche Grenze lässt sich durch die Niedriglohnschwelle definieren, die üblicherweise mit zwei Drittel des Medianlohns von Vollzeitbeschäftigten angegeben wird (OECD 2006, S. 174ff.).

(2) Beschäftigungsstabilität: Mit Beschäftigungsstabilität ist nicht die Sicherung eines gegebenen spezifischen Arbeitsplatzes (job security) gemeint, sondern bei Strukturwandel die Sicherstellung möglichst nahtloser Beschäftigungsverhältnisse. Sie ist Voraussetzung für ein eigenständiges Einkommen sowie für ausreichende Ansprüche an die sozialen Sicherungssysteme.

(3) Beschäftigungsfähigkeit: Beschäftigungsfähigkeit (employability) dient bei raschem Strukturwandel und dadurch verursachten Änderungen in den Qualifikationen und Tätigkeiten als Voraussetzung für Beschäftigungsstabilität. Eine für die Beschäftigungsfähigkeit zentrale Voraussetzung ist lebenslanges Lernen. Als Indikator bietet sich die Teilnahme an beruflicher Weiterbildung an.

(4) Integration in soziale Sicherungssysteme: Soziale Sicherheit schließt die ausreichende materielle Absicherung gegenüber den sozialen Risiken infolge Krankheit, Arbeitslosigkeit und Alter durch die sozialen Sicherungssysteme ein. Da die in der nachfolgenden empirischen Analyse verwendeten Daten der Vierten Europäischen Umfrage über Arbeitsbedingungen der Europäischen Stiftung von 2005 (European Foundation 2007a) dieses Kriterium nicht unseren Anforderungen entsprechend abbilden, bleibt es in der weiteren Analyse unberücksichtigt.

Mithilfe dieser Kriterien lassen sich Dimensionen von Prekarität (wie beispielsweise Einkommens- oder Beschäftigungsprekarität) angeben. Soziale Sicherungsstandards gelten als prekär, wenn be- stimmte Schwellenwerte nicht erreicht oder Tatbestände (z. B. Teilnahme an beruflicher Weiterbildung) nicht erfüllt werden. Während sich beim Einkommen mit dem oben definierten Wert für ein Niedrigbzw. Prekaritätseinkommen ein Schwellenwert spezifizieren lässt, bereitet ein analoges Vorgehen bei den anderen Dimensionen erhebliche Probleme. Relativierend ist anzumerken, dass die Abgrenzungen zwischen einem als prekär und einem als nicht prekär klassifizierten Status fließend bleiben, solange nicht weitere Fragen geklärt sind. Denn erstens hängt der Prekaritätsgrad von der Dauer ab, mit der ein Beschäftigungsverhältnis unter Bedingungen unzureichender sozialer Sicherung ausgeübt wird. Grundsätzlich ist von einer engen Korrelation zwischen beiden Größen auszugehen. Wird beispielsweise ein nur kurzzeitig ausgeübtes befristetes Beschäftigungsverhältnis entfristet oder dient Leiharbeit als Brücke aus Arbeitslosigkeit in ein Normalarbeitsverhältnis mit einer über dem Prekaritätslohn liegenden Vergütung, ${ }^{5}$ bleibt der Prekaritätsgrad marginal. Zweitens spielt die Frage der freiwilligen Wahl einer bestimmten Beschäftigungsform eine Rolle. Denkbar ist, dass Beschäftigte aus Gründen der beruflichen Orientierung und Qualifizierung für ein befristetes Beschäftigungsverhältnis optieren oder sich aus anderen (privaten oder familiären) Gründen für eine phasenweise Teilzeitbeschäftigung entscheiden. Bedeutsam ist drittens die Unterscheidung zwischen Individual- und Haushaltsperspektive, wenn es um die Bewertung der Einkommenshöhe geht. Die nachfolgenden Analysen beziehen sich allein auf die Individualebene. Datenbedingt ist eine weitere Restriktion der Einkommensdimension: Ausgeklammert bleibt die Höhe der Subsistenzleistungen im Fall des Arbeitsplatzverlustes sowie der Transferzahlungen in der Nacherwerbsphase. Ebenso wenig gestatten die verfügbaren Daten, die Kriterien der Freiwilligkeit und der Dauer des prekären $\mathrm{Zu}$ standes differenziert und angemessen zu berücksichtigen. Angesichts dieser Einschränkungen lassen sich mit Hilfe von Indikatoren relative Werte für Prekarität berechnen, die ermittelten Prekaritätsgrade international vergleichen und in Beziehung zu Flexibilitätsgraden setzen.

\section{Daten und Methode}

Der Zusammenhang von Flexibilität und sozialer Sicherheit bzw. Prekarität wird mithilfe der in Abschnitt 2 beschriebenen Dimensionen und Indikatoren analysiert. Deren Operationalisierung stützt sich, wie bereits erwähnt, auf Daten der Vierten Europäischen Umfrage über Arbeitsbedingungen der Europäischen Stiftung. Dabei handelt es sich um eine im Jahr 2005 in 31 Ländern durchgeführte Personenerhebung, die ein breites Themenspektrum zu verschiedenen Aspekten der Arbeitsbedingungen umfasst. Unsere Analyse bleibt auf die 27 EU-Mitgliedsländer beschränkt; sie basiert auf Antworten von 20.841 abhängig Beschäftigten der insgesamt 29.860 Befragten.

Die Datenstruktur lässt sich als eine große Tabelle darstellen, wobei jede Zeile die Antworten einer Person zu 34 ausgewählten Fragen enthält: davon beziehen sich 21 auf die vier Dimensionen der Flexibilität und 13 auf die drei Dimensionen der sozialen Sicherheit bzw. Prekarität. ${ }^{6}$ Jede Spalte mit kodierten Antworten von 20.841 Personen zu den einzelnen Fragen wird als Variable betrachtet. Alle Variablen werden umkodiert: je höher der Wert, desto flexibler, bzw. prekärer ist die Arbeitssituation (Details s. Tangian 2007b). Im nächsten Schritt werden die umkodierten Variablen nach zwei Methoden skaliert: Die in der Hans-Böckler-Stiftung entwickelte Methode basiert auf einer Normalisierung der Variablen (Tangian 2007a; Tangian 2007c), die der OECD auf einer Standardisierung der Variablen (OECD-JRC 2005). Die zusammengesetzten Indikatoren sind entsprechend ungewichtet oder gewichtet. Die beiden Methoden führen zu einem sehr ähnlichen Benchmarking der Länder ( $A b$ bildungen 1 und 2). Dieser Tatbestand zeigt, dass die Ergebnisse ziemlich robust sind, da sie wenig von der Gewichtung und von der Methode abhängen.

\footnotetext{
5 Leiharbeitnehmer, die in ein Normalarbeitsverhältnis wechseln, haben im Vergleich zu Beschäftigten, die niemals in Zeitarbeit tätig waren, langfristig keine Lohneinbußen (Kvasnicka/Werwatz 2003).

6 Zu der Liste der Variablen und ihrer Gruppierung vgl. Seifert/Tangian 2007.
} 
Die Normalisierung (HBS-Methode, Abbildung 1) reduziert die theoretisch erreichbaren absoluten min- und max-Werte jeder Variablen auf 0 und 1 (wenn alle Befragten extrem negativ bzw. extrem positiv eine Frage beantworten). Die Variable wird dann in Prozent ihrer Spannbreite ausgedrückt. Dafür wird jede Variable $x=$ $\left(x_{1}, \ldots, x_{n}\right)^{\prime}, n=20.841$, wie folgt umgeformt:

$$
y=\frac{x-x_{\min }}{x_{\max }-x_{\min }} \cdot 100 \%
$$

(normalisierte Variable in \%)

Die Standardisierung (OECD-Methode) reduziert den Mittelwert und die Standardabweichung jeder Variable auf 0 und 1 . Zusätzlich wird die Variable in Prozent ihrer Standardabweichung ausgedrückt:

$$
y=\frac{x-\mu}{\sigma} \cdot 100 \%
$$

(standardisierte Variable in \%)

Der 0-Wert von $y$ entspricht dem Mittelwert von $x$, und 100 entspricht der Standardabweichung von $x$ :

$$
\begin{aligned}
& \mu=\frac{1}{n} \sum_{i=1}^{n} x_{i} \text { (empirischer Mittelwert) } \\
& \sigma=\sqrt{\frac{1}{n-1} \sum_{i=1}^{n}\left(x_{i}-\mu\right)^{2}}
\end{aligned}
$$

Die Normalisierung drückt die Variablen in einer absoluten Skala aus, sodass man zwischen „guten“ und „schlechten“ Werten differenzieren kann. Die Standardisierung relativiert die "guten“ und „schlechten“ Werte, indem sie die Abweichungen von dem Durchschnittswert indiziert. Normalisierung eignet sich eher für Evaluierungen; und Standardisierung eher für Benchmarking. Wir wenden beide Methoden an und vergleichen die Ergebnisse. Unabhängig von der Methode werden die Antworten jeder Person in Gruppen summiert, um die aggregierten Antworten jeder Person für vier Flexibilitäts- und drei Prekaritätsformen zu berechnen. Die OECD weist darauf hin (OECD-JRC 2005, S. 21), dass die meisten zusammengesetzten Indikatoren auf der gleichen Gewichtung basieren, d.h. alle Variablen erhalten gleiche Gewichte. Die hier durchgeführten Analysen folgen diesem Prinzip. Allerdings ändert die Standardisierung die effektive Spannbreite der Variablen und führt deswegen eine $\mathrm{Ge}$ wichtung ein, wobei die Gewichte in einem umgekehrt proportionalen Verhältnis zu

\section{Abb. 1: Indikatoren der Flexibilität und Prekarität für EU-27 (HBS-Methode)}

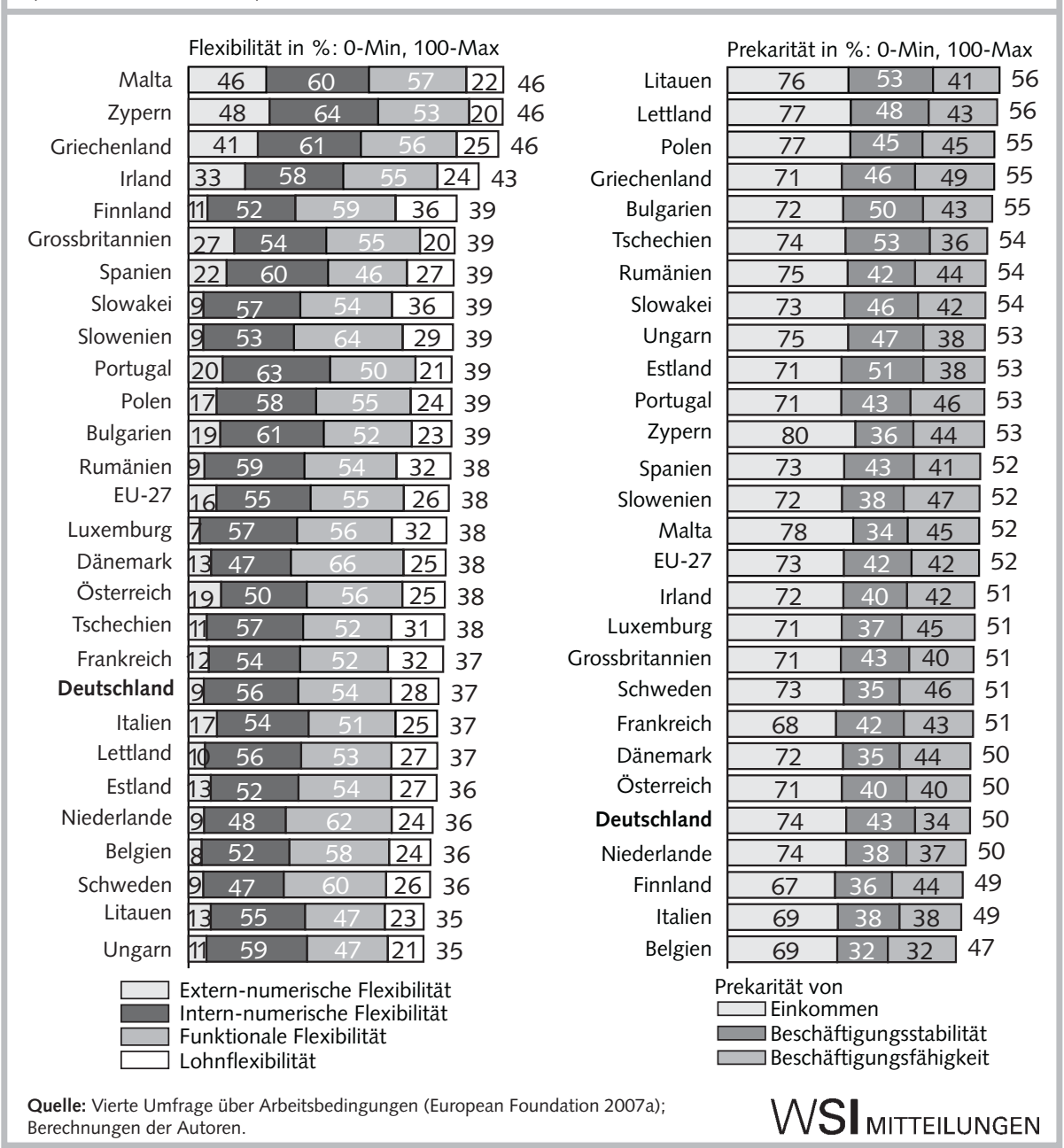

den Standardabweichungen der Variablen stehen.

Jeder der sieben erststufig aggregierten Indikatoren wird entweder normalisiert (HBS-Methode) oder standardisiert (OECD-Methode). Nach dem gleichen Muster werden auch die in der nächsten Stufe aggregierten Gesamtindikatoren für Flexibilität und für Prekarität berechnet.

Die erst- und zweitstufig aggregierten Indikatoren sind wie folgt $\mathrm{zu}$ interpretieren: Nach der HBS-Methode drückt der aggregierte Index einer Person die durchschnittlichen Antworten der Person auf entsprechende Fragen aus. Die Werte $0 \%$ oder $100 \%$ werden erreicht, wenn alle Fragen extrem beantwortet werden. Nach der OECD-Methode stellt ein zusammengesetzter Indikator die gewichtete Summe der Variablen dar, wobei die Gewichte nach den Varianzen der Variablen ermittelt werden. Bei mehrstufigen Aggregationen kommt es zu ungewöhnlichen Effek- ten: Größere erststufig aggregierte Indikatoren können in der nächsten Aggregationsstufe kleinere Werte annehmen und umgekehrt.

Die Unterschiede in den Berechnungen nach beiden Methoden bringen die Abbildungen 1 und 2 zum Ausdruck. Die Beiträge der erststufig aggregierten Indikatoren zu Gesamtindikatoren werden durch Balken angezeigt. In Abbildung 1 (HBS-Methode) ist der Einfluss der extern-numerischen Flexibilität auf den gesamten Indikator (Werte rechts vom Balken) deutlich kleiner als der der intern-numerischen Flexibilität. Da die OECD-Methode die Beiträge relativiert, werden die Einflüsse aller Dimensionen in Abbildung 2 angeglichen.

Die Länder sind nach den fallenden Gesamtindikatoren (Werte rechts vom Balken) geordnet. Für die HBS-Methode sind die Gesamtindikatoren proportional zur gesamten Balkenlänge, was bei der OECDMethode nicht der Fall ist. Beispielsweise 


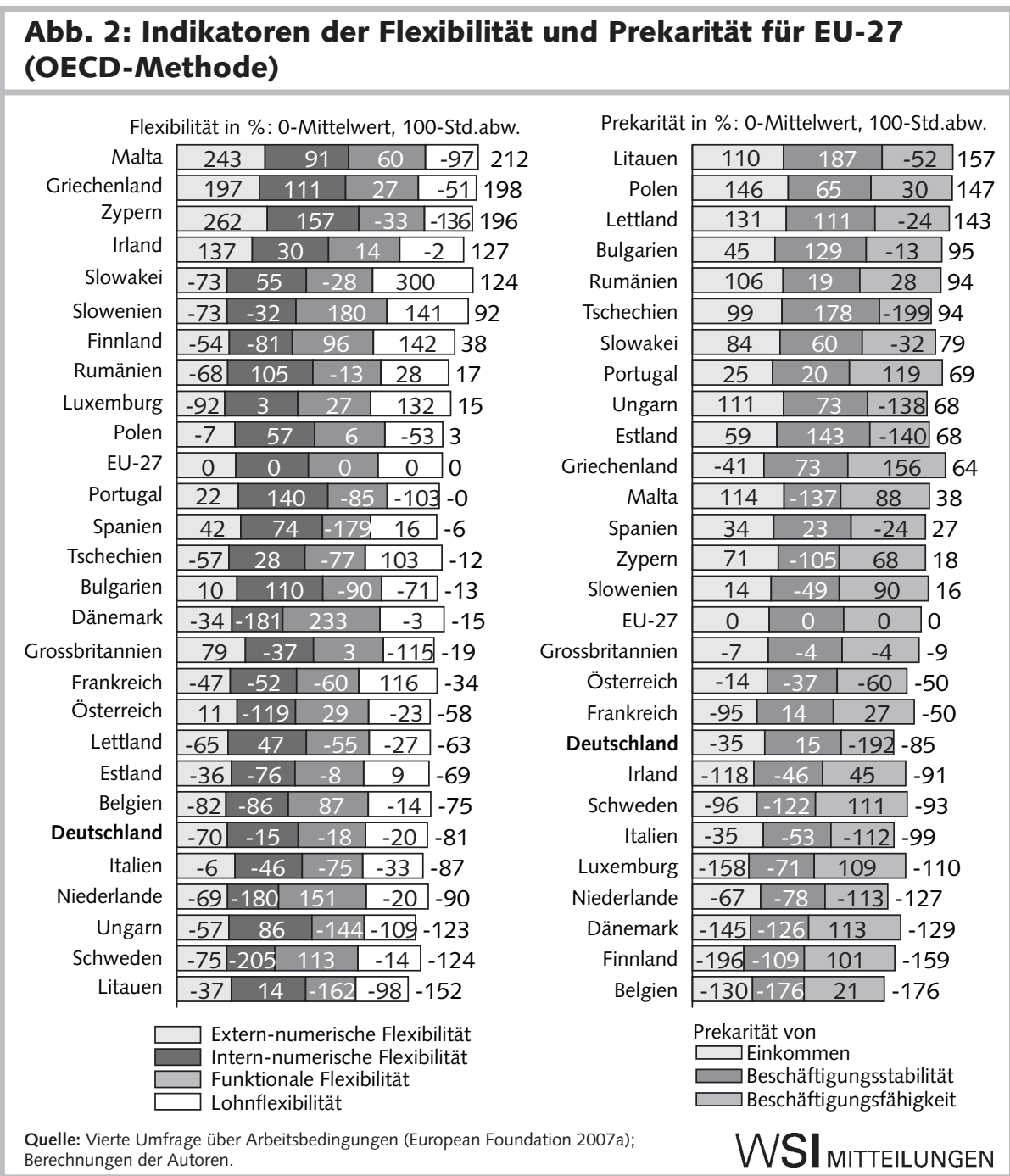

ist der aggregierte Gesamtwert (Gesamtbalken) für Irland in der 1 . Spalte der Abbildung 2 kleiner als der für die Slowakei, obwohl der Grad der Flexibilität im ersten Land (127) größer ist als im zweiten (124). Diese Eigentümlichkeit resultiert als Nebeneffekt aus der mehrstufigen Standardisierung. Der Vorteil der OECD-Methode ist darin zu sehen, dass die Abweichungen vom Durchschnitt $(=0)$ sofort sichtbar werden. und 2), zeigt folgendes Bild: Der Gesamtindikator (HBS-Methode) bewegt sich für alle Länder in der unteren Hälfte der Skala. Ausgespart bleiben die Bereiche extrem hoher und extrem geringer Flexibilität. Die OECD-Methode zieht die Länderwerte weiter auseinander, lässt sie weniger gestaucht erscheinen. Beide Methoden ordnen die betrachteten Länder nach einer ähnlichen Rangfolge, was der hohe Rangkorrelationskoeffizient ( $\varrho=0,92)$ belegt. Auch für die Teilindikatoren zeigen beide Berechnungsmethoden ein weitgehend übereinstimmendes Bild, das die hohen Werte der Koeffizienten der Rangkorrelation bestätigen (Tabelle 1).

Deutschland rangiert bei der Flexibilität, gemessen am Gesamtindikator, im unteren Mittelfeld. Allerdings sind die Abstände zum EU-Durchschnittswert oder zu Plätzen im oberen Mittelfeld nur gering.

Betrachtet man die einzelnen Flexibilitätsdimensionen, so zeigen sich beträchtliche Unterschiede im Flexibilitätsgrad. Intern-numerische und funktionale Flexibilität erreichen in allen Ländern deutlich höhere Werte als extern-numerische oder Lohnflexibilität. In den meisten Ländern erzielt der Teilindikator für monetäre Flexibilität höhere Werte als der für externnumerische. Auf die bedeutende Rolle, die Formen intern-numerischer Flexibilität in betrieblichen Anpassungsstrategien spielen, weisen auch Analysen der EU-Kommission hin, so eine Betriebsbefragung (European Commission 2001) sowie die Analysen über intern-numerische und funktionale Flexibilität (European Commission 2007b).

Die hohen Teilindizes für intern-numerische und intern-funktionale Flexibilität spiegeln ein komplementäres Verhältnis der beiden Dimensionen. Wie Fallstudien zeigen, haben Verkürzungen der Arbeitszeit, die der Anpassung an einen reduzierten Arbeitseinsatz dienen, organisatorische Umstrukturierungen und betriebs- bzw. unternehmensinterne Umsetzungen zur Folge (Promberger et al. 1996). Die unterschiedliche Bedeutung von interner und externer Flexibilität kann damit zu tun haben, dass beide Dimensionen in einem substitutiven Verhältnis zueinander stehen und die erstgenannte Anpassungsform je nach den betrieblichen Strukturen und den Anpassungserfordernissen Kostenvorteile sowohl für Betriebe als auch Beschäftigte bietet (Seifert/Massa-Wirth 2005). 
Für Deutschland weichen die Werte der Einzelindikatoren nur wenig von den Durchschnittswerten der EU ab. Externnumerische Flexibilität spielt eine geringere Rolle, die übrigen Flexibilitätsdimensionen liegen leicht über den EU-Werten.

Der Ländervergleich offenbart unterschiedlich komponierte Gesamtindikatoren. Gleiche Werte bei den nationalen Gesamtindikatoren können durch unterschiedlich zusammengesetzte Flexibilitätsformen zustande kommen. So sind beispielsweise die Werte von Spanien und Slowenien für die aggregierte Flexibilität identisch, die einzelnen Flexibilitätsformen gehen jedoch mit einem unterschiedlichen Gewicht in den Gesamtindikator ein. In Spanien erreicht extern-numerische Flexibilität einen wesentlich höheren Wert, in Slowenien dagegen funktionale Flexibilität. Offensichtlich führen verschiedene Wege nach Rom bzw. zu hoher Gesamtflexibilität. Es muss weiteren empirischen Analysen überlassen bleiben, die Hintergründe für die unterschiedlichen nationalen Flexibilitätsmuster aufzuhellen.

Nur geringe Übereinstimmung zeigen die hier (nach beiden Methoden) für die faktische Flexibilität berechneten Ergebnisse mit den Werten der EPL-Evaluierung der OECD, die den Flexibilitätsgrad auf Basis der institutionell-rechtlichen Normen indizieren. So rangiert nach unseren Schätzungen beispielsweise der Teilindikator der externen Flexibilität für Griechenland im europäischen Vergleich sehr hoch, während der EPL-Indikator der OECD die externe Flexibilität als relativ gering einstuft (OECD 2004, S. 117). Dieser Unterschied hat vor allem damit zu tun, dass nach den hier verwendeten Daten $32 \%$ der in Griechenland Beschäftigten ohne Arbeitsvertrag arbeiten, also in extrem flexibler Form beschäftigt sind. Flexibilität findet hier jenseits des rechtlichen Rahmens statt. Derartige Tatbestände entziehen sich der Analyse des institutionell-rechtlichen Rahmens von Flexibilität. Deren Ergebnisse können deshalb erheblich von denen der faktischen Flexibilität abweichen.

Die Werte für die Indikatoren der einzelnen Flexibilitätskomponenten streuen teilweise stark zwischen den EU-Mitgliedsländern. Besonders weit klaffen sie auseinander für extern-numerische Flexibilität, die für Zypern das höchste und für Luxemburg das niedrigste Niveau aufweist. Auf den ersten Blick überraschend erscheint der niedrige Indikator für Dänemark. Er könn-

\section{Abb. 3: Zusammenhang der aggregierten Flexibilitäts- und Prekaritäts- indikatoren (HBS-Methode)}

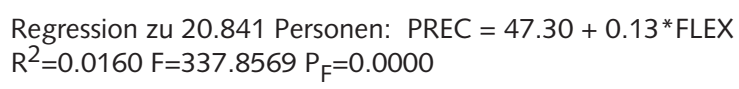

Regression zu 20.841 Personen: PREC $=47.30+0.13^{*}$ FLEX

$R^{2}=0.0160 F=337.8569 P_{F}=0.0000$

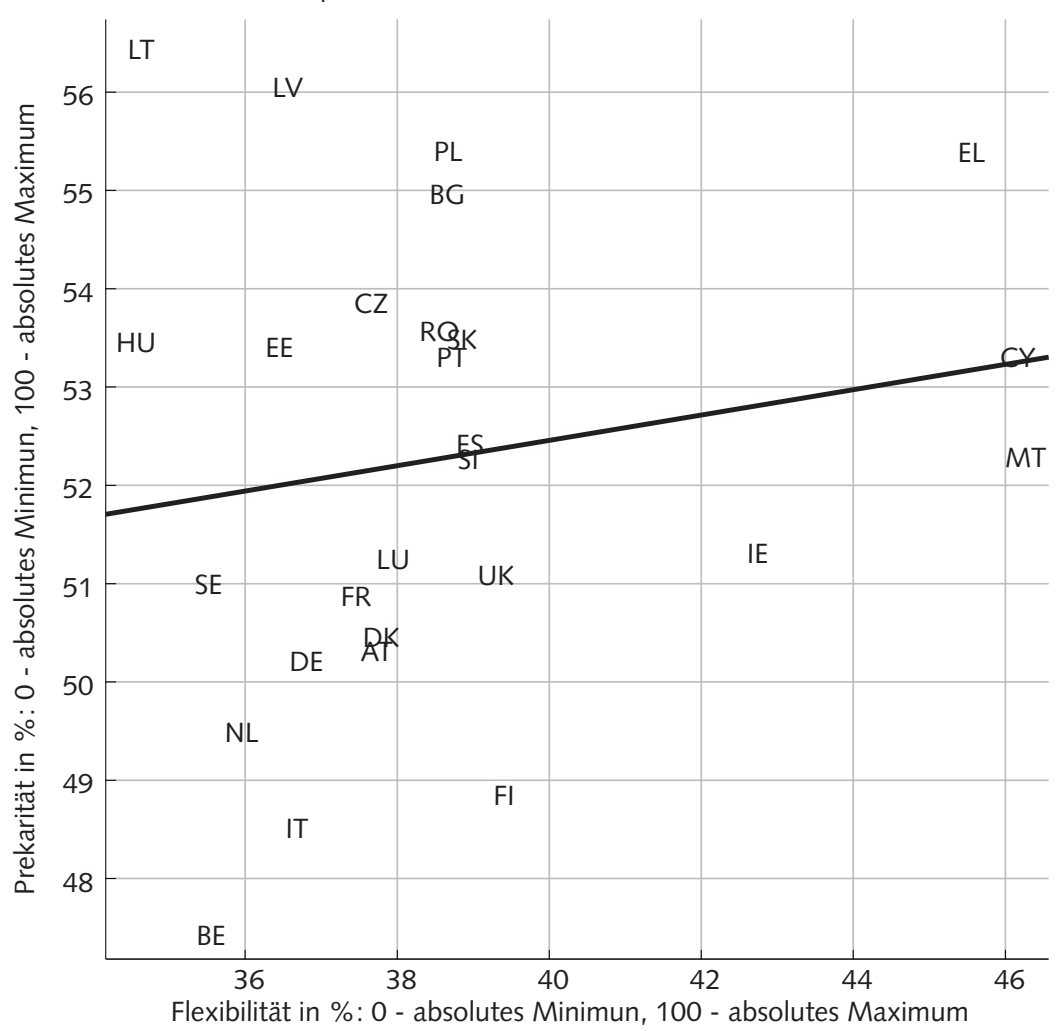

BE-Belgien, CZ-Tschechien, DK-Dänemark, DE-Deutschland, EE-Estland, EL-Griechenland, ES-Spanien, FR-Frankreich, IE-Irland, IT-Italien, CY-Zypern, LV-Lettland, LT-Litauen, LU-Luxemburg, HU-Ungarn, MT-Malta, NL-Niederlande, ATÖsterreich, PL-Polen, PT-Portugal, SI-Slowenien, SK-Slowakei, FI-Finnland, SE-Schweden, UK-Großbritannien, BGBulgarien, RO-Rumänien

Quelle: Vierte Umfrage über Arbeitsbedingungen (European Foundation 2007a); Berechnungen der Autoren.

te damit zu tun haben, dass der relativ geringe gesetzliche Kündigungsschutz bei unbefristeten Arbeitsverhältnissen wenig Anlass bietet, den von den Betrieben benötigten Flexibilitätsbedarf über befristete Arbeitsverhältnisse und Leiharbeit zu organisieren. Gleichzeitig erzielt der Indikator für funktionale Flexibilität in Dänemark den höchsten Wert unter den EU-Mitgliedsländern. Er ist auf eine hohe Weiterbildungsintensität und eine flexible Arbeitsorganisation zurückzuführen. Über dieses Einzelbeispiel hinausgehend muss an dieser Stelle die Frage offen bleiben, wie die beobachteten Unterschiede in den Flexibilitätsmustern zu erklären sind, welche Rolle rechtlich-institutionelle Faktoren, die Wirtschaftsstrukturen, die industriellen Beziehungen oder auch öffentliche Förderpolitiken spielen.

\subsection{PREKARITÄT}

Der Indikator, der die soziale Sicherheit bzw. Prekarität der Arbeit misst, bewegt sich in den Mitgliedsländern der EU in einem mittleren Bereich der Skala (HBSMethode, Abbildung 1). Er weist für kein Land Extremwerte aus; weder das oberste noch das unterste Drittel der Skala sind besetzt. Die nach beiden Methoden berechneten Prekaritätsindikatoren (Tabelle 1) ordnen die EU-Länder nach einer weitgehend übereinstimmenden Rangfolge (Rangkorrelationskoeffizient $\varrho=0,93$ ).

Der Wert für Deutschland liegt im unteren Drittel auf dem gleichen Niveau wie die Werte für Dänemark, Österreich und die Niederlande. Demgegenüber weisen die Werte für Litauen, Lettland und Polen auf ein insgesamt (gemessen an den drei Teilindikatoren) vergleichsweise hohes Prekaritätsniveau hin.

Unter den Prekaritätskomponenten erzielt der Partialindikator für die Einkommensprekarität in allen Ländern die höchsten Werte. Der in Deutschland relativ hohe, über dem EU-Durchschnitt liegende Wert für Einkommensprekarität über- 


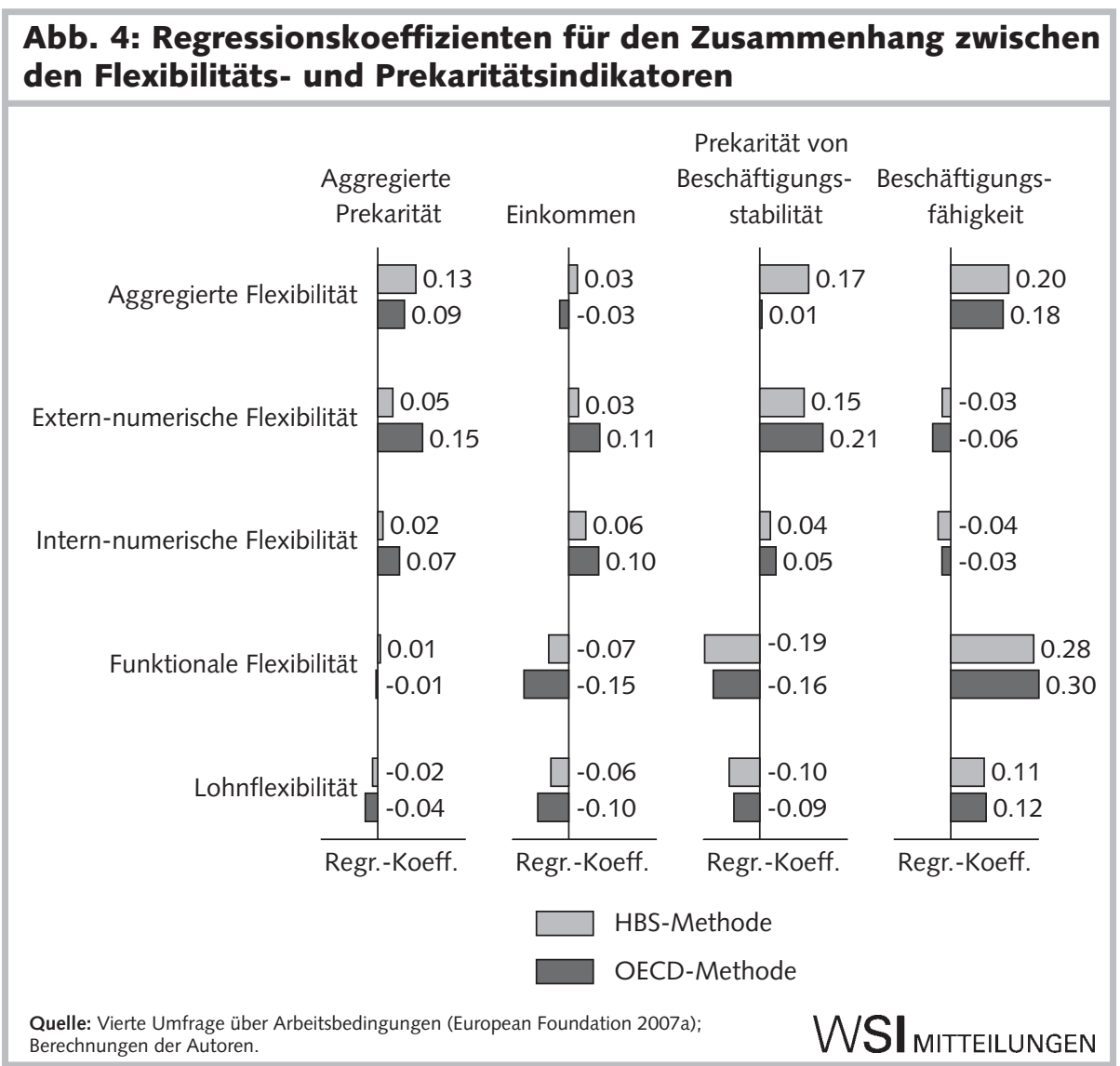

rascht nicht, da hier der Niedriglohnsektor in den letzten Jahren stärker als in anderen Ländern expandierte (Kalina/Weinkopf 2008). Bei den Teilindikatoren der Beschäftigungsstabilität und Beschäftigungsfähigkeit liegen die Prekaritätswerte auf einem deutlich niedrigeren Niveau. Bei der Beschäftigungsfähigkeit schneidet Deutschland relativ am besten ab. Dieses Ergebnis überrascht, da in den Indikator neben den Karriereperspektiven, der angenommenen Fähigkeit, die derzeitige Arbeit auch in einem Alter von 60 und mehr Jahren ausüben zu können, auch die Teilnahme an der beruflichen Weiterbildung eingeht, die in Deutschland im europäischen Vergleich aber nur durchschnittlich ist (Behringer et al. 2008).

\subsection{NEXUS FLEXIBILITÄT - PREKARITÄT}

Zwischen Flexibilität und Prekarität besteht statistisch ein positiver, allerdings nicht allzu starker Zusammenhang. Die mit der HBS-Methode bestimmte Regressionslinie (Abbildung 3) zeigt, dass der Prekaritätsgrad mit der Flexibilisierung steigt. Der kleine $\mathrm{R}^{2}$-Wert bedeutet, dass die individuellen Beobachtungen nicht entlang der
Werte ein im internationalen Vergleich mittleres Niveau der Flexibilität bei vergleichsweise niedriger Prekarität aus.

\subsection{ZUSAMMENHANG DER EINZELINDIKATOREN}

Abbildung 4 gibt die Regressionskoeffizienten für die aggregierten sowie die Teilindikatoren für Flexibilität und Prekarität in den 27 EU-Mitgliedsländern wider. Die oberen Balken indizieren die mit der HBSMethode berechneten Werte, die unteren Balken die mit der OECD-Methode gewonnenen. Beispielsweise weist das obere linke Balkenpaar, das den Zusammenhang zwischen aggregierter Flexibilität und aggregierter Prekarität indiziert, Regressionskoeffizienten von 0.13 und 0.09 auf. Auch für die Teilindikatoren kommen die Berechnungen nach beiden Methoden zu ähnlich übereinstimmenden Ergebnissen:

(1) Zwischen extern-numerischer Flexibilität und Einkommen sowie Beschäftigungsstabilität besteht ein negativer $\mathrm{Zu}$ sammenhang. Der Prekaritätsgrad steigt mit externer Flexibilität. Dieser Zusammenhang dürfte damit zu tun haben, dass der Indikator der extern-numerischen Flexibilität weitgehend durch die Anteilswerte atypischer Beschäftigung (Befristung, Leiharbeit, Dauer der Arbeitszeit) gebildet wird. Diese Formen des Erwerbsstatus bergen ein hohes Risiko, ein geringeres Einkommen als bei Normalarbeit zu erzielen und zudem instabiler beschäftigt zu sein (OECD 2006).

(2) Für den Zusammenhang zwischen intern-numerischer Flexibilität und Prekarität zeigt sich - allerdings abgeschwächt ein ähnliches Bild. Der Einkommenseffekt ist plausibel, da Reduzierungen der Arbeitszeit nicht einkommensneutral sind, sondern proportionale Abstriche bedeuten. Nicht plausibel ist dagegen der negative Zusammenhang mit der Beschäftigungsstabilität.

(3) Mit dem Grad der funktionalen Flexibilität sinkt der Grad der Einkommensund der Beschäftigungsprekarität, und umgekehrt steigt der der Beschäftigungsfähigkeit. Der letztgenannte Effekt überrascht und könnte damit zu tun haben, dass Beschäftigte mit relativ geringer Beschäftigungsfähigkeit und schwacher Arbeitsmarktposition einem stärkeren Druck 
ausgesetzt sind, unterwertige, aber noch als zumutbar eingestufte Arbeit zu akzeptieren und häufiger Tätigkeitswechsel vorzunehmen. Der erstgenannte Zusammenhang könnte darauf zurückzuführen sein, dass funktionale Flexibilität in Situationen, in denen Betriebe Arbeitskräfte abbauen, bessere Chancen für einen Verbleib auf dem internen Arbeitsmarkt eröffnet. Breite Qualifikationen und Teilnahme an beruflichen Weiterbildungen, zwei zentrale Variablen für diesen Indikator, können außerdem höhere Löhne begründen.

(4) Lohnflexibilität zeigt in der Richtung den gleichen Zusammenhang wie funktionale Flexibilität, allerdings deuten die Regressionskoeffizienten auf einen geringeren Wirkungszusammenhang. Dieses Ergebnis lässt sich so interpretieren, dass Arbeitnehmer mit niedriger Beschäftigungsfähigkeit eher variabel bezahlte Arbeit akzeptieren.

Da die Regressionskoeffizienten in standardisierten Variablen Korrelationskoeffizienten darstellen, zeigen die mit der standardisierenden OECD-Methode berechneten Werte die Korrelation zwischen den Indikatoren. Die Korrelation ist nicht groß, aber statistisch signifikant. lität, sondern auch von der Struktur der Flexibilitätsformen ab. Diese Zusammenhänge sind in weiteren Forschungsarbeiten näher auszuleuchten. Forschungsbedarf besteht auch bei der Frage, wie die zwischen den EU-Ländern beobachteten Streuungen bei den Werten sowohl für Flexibilität als auch Prekarität zu erklären sind.

Für die weitere politische Diskussion über Flexicurity verweisen die Befunde der vorliegenden Untersuchung vor allem auf die im engen Kontext mit Flexibilität beobachteten Prekaritätsprobleme. Flexibilität am Arbeitsmarkt geht offensichtlich auf Kosten der sozialen Sicherheit. Das Augenmerk der nationalen Flexicurity-Konzepte sollte deshalb darauf gerichtet sein, die negativen Effekte der Flexibilität zu kompensieren. Ungeachtet der länderspezifischen Besonderheiten geht es generell um die Gleichbehandlung sämtlicher Beschäftigungsformen bei Lohn, Zugang zu beruflicher Weiterbildung und Integration in die sozialen Sicherungssysteme, ferner um arbeitsmarktpolitische Hilfestellungen bei Beschäftigungsübergängen sowie eine ausreichende Grundsicherung in der Nacherwerbsphase. Verbleiben dennoch besondere Prekaritätsprobleme, bieten Risikoprämien einen Weg der Kompensation. men erreichen lassen. Offensichtlich bestehen substitutive Verhältnisse zwischen den Formen der Flexibilität, vor allem zwischen internen und externen Varianten.

Der Grad der Gesamtprekarität hängt nicht nur vom Ausmaß der Gesamtflexibi- 
Atkinson, J. (1984): Flexibility, Uncertainty and Manpower Management, Brigthon

Behringer, F./Moraal, D./Schönfeld, G. (2008): Betriebliche Weiterbildung in Europa: Deutschland weiterhin nur im Mittelfeld: Aktuelle Ergebnisse aus CVTS 3, in: Berufsbildung in Wissenschaft und Praxis 37, S. 9-14 Brinkmann, U./Dörre, K./Röbenack, S. (2006): Prekäre Arbeit. Ursachen, Ausmaß, soziale Folgen und subjektive Verarbeitungsformen unsicherer Beschäftigungsverhältnisse, Bonn

Edwards, R. C./Reich, M./Gordon, D. M. (Hrsg.) (1973): Labor Market Segmentation, Lexington/Mass., Toronto, London

European Commission (2001): Performance of the European Union labour market. Joint harmonised EU programme of business and consumer surveys, Luxembourg

European Commission (2006a): Green Paper. Modernising labour law to meet the challenges of the $21^{\text {st }}$ century, Brussels

European Commission (2006b): Employment in Europe, Luxembourg European Commission (2007a): Towards Common Principles of Flexicurity: More and better jobs through flexibility and security, Luxembourg European Commission (2007b): Employment in Europe, Luxembourg European Commission (2007c): Ten years of the European Employment Strategy (EES), Luxembourg

European Foundation for the Improvement of Working and Living Conditions (2003): Flexibility and Social Protection, Dublin European Foundation for the Improvement of Working and Living Conditions (2007a): Fourth European Working Conditions Survey, Dublin European Foundation for the Improvement of Working and Living Conditions (2007b): Approaches to Flexicurity: EU Models, Dublin Kalina, T./Weinkopf, C. (2008): Weitere Zunahme der Niedriglohnbeschäftigung: 2006 bereits rund 6,5 Millionen Beschäftigte betroffen, in: IAQ-Report 2008-01, Essen

Kvasnicka, M./Werwatz, A. (2003): Lohneffekte der Zeitarbeit, in: Bundesarbeitsblatt 2, S. 2-10

Keller, B./Seifert, H. (Hrsg.) (2007): Atypische Beschäftigung - Flexibilisierung und soziale Risiken, Berlin

Keller, B./Seifert, H. (2008): Flexicurity: Ein europäisches Konzept und seine nationale Umsetzung, Friedrich-Ebert-Stiftung, Bonn
Kraemer, K. (2008): Prekarität - was ist das?, in: Arbeit 17, S. 77-90 OECD (1986): Flexibility in the labour market. The current debate, Paris OECD (1999): Employment outlook, Paris OECD (2004): Employment outlook, Paris OECD (2006): Employment outlook, Paris OECD-JRC (2005): Handbook on Constructing Composite Indicators: Methodology and User Guide, Paris

Promberger, M./Rosdücher, J./Seifert, H./Trinczek, R. (1996): Beschäftigungssicherung durch Arbeitszeitverkürzung, Berlin

Rodgers, G. (1989): Precarious work in Western Europe: The state of the debate, in: Rodgers, G./Rodgers, J. (Hrsg.): Precarious Jobs in Labour Market Regulation, Geneva, S. 1-16

Salengros, P./van de Leemput, C./ Mubikangiey, L. (1989): Psychological and sociological perspectives on precarious employment in Belgium, in: Rodgers, G./Rodgers, J. (Hrsg.): Precarious Jobs in Labour Market Regulation, Geneva, S. 197-224

Seifert, H./Massa-Wirth, H. (2005): Pacts for employment and competitiveness in Germany, in: Industrial Relations Journal 36, S. 217-240 Seifert, H./Tangian, A. (2007): Reconciling social security with flexibility empirical findings for Europe, WSI-Diskussionspapier 154, Hans-BöcklerStiftung, Düsseldorf

Tangian A. (2007a): Analysis of the third European survey on working conditions with composite indicators, European Journal of Operational Research 181, S. 468-499

Tangian, A. (2007b): Is flexible work precarious? A study based on the 4th European survey of working conditions 2005, WSI-Diskussionspapier 153, Hans-Böckler- Stiftung, Düsseldorf

Tangian, A. (2007c): European flexicurity: concepts, methodology and politics, in: Transfer 13, S. 551-574

Wilthagen, T. (rapporteur) (2007): Flexicurity pathways. Expert group on flexicurity, Interim report, Brussels

Wilthagen, T./Tros, F. (2004): The concept of "flexicurity": A new approach to regulating employment and labour markets, in: Transfer 10, S. $166-186$ 